\title{
Myofibroblasts in hepatitis B related cirrhosis and hepatocellular carcinoma
}

\author{
K Y Chau, M A Lily, P C Wu, W L Yau
}

\begin{abstract}
Peritoneal liver biopsy specimens from eight patients with hepatitis $B$ associated cirrhosis, complicated by hepatocellular carcinoma, were studied for identification and localisation of myofibroblasts. The avidin-biotin peroxidase complex technique was used on paraffin wax sections, using monoclonal antibodies for actin and desmin, and ultrastructural examination was performed. Myofibroblasts were found in seven of the eight cirrhotic specimens and in all eight tumour specimens. They were identified in the fibrotic areas by the immunohistochemical technique, but ultrastructural examination disclosed their presence in the perisinusoidal space and between tumour cells.
\end{abstract}

Myofibroblasts are transformed fibroblasts with contractile properties. They are found in normal tissue, repair tissue, and a variety of benign, borderline, and malignant fibrous or fibrohistiocytic tumours. Their prevalence and role in the desmoplastic reaction of tumours were examined recently. ${ }^{12}$

\section{Methods}

Immunohistochemical and ultrastructural studies were performed on biopsy specimens from eight patients with hepatitis B associated cirrhosis, complicated by hepatocellular carcinoma. The biopsy specimens were taken both from cirrhotic areas and tumours using a peritoneoscope. Each specimen was fixed in $10 \%$ formalin immediately after biopsy. They were then divided into two portions and processed for histological and ultrastructural studies. These patients were either seropositive for hepatitis B surface antigen or their liver biopsy specimens showed ground-glass hepatocytes which stained positively for aldehyde, fuchsin, or both. No other causes for cirrhosis were identified.

An avidin-biotin peroxidase complex technique with monoclonal antibodies specific for actin (HHF 35; Biogenex and desmin D 33; Dakopatts) were used to identify myofibroblasts in paraffin wax sections. Both myofibroblasts and smooth muscle cells express actin ${ }^{3}$ and, also weakly, desmin. Electron microscopic examination was also performed on all the specimens to identify myofibroblasts. They were characterised ultrastructurally by peripherally located cyto- plasmic actin filaments with dense bodies, in an otherwise typical fibroblast containing prominent rough endoplasmic reticulum, Golgi apparatus, and an indented nucleus.

\section{Results}

Histologically, besides perivascular smooth muscle cells, myofibroblasts expressing actin and, weakly, desmin, were identified. Five out of the eight cirrhotic liver specimens showed occasional numbers of these cells in the fibrous septa; none of these cells was identifiable in the perisinusoidal space using immunohistochemistry. In seven of the eight tumour specimens scanty to moderate numbers of these cells were seen in the fibrous tissue surrounding or separating tumour masses, close to the margins of the tumour islands (fig 1). They were moderate in number in one case and occasional or scanty in six cases. The negative one contained only tumour cells arranged in thick trabeculae showing no fibrous stroma. Positive staining cells were not identified within the tumour masses in any of the cases.

Ultrastructurally, myofibroblasts were found in five of the eight cirrhotic specimens, including two immunohistochemically negative cases. They were also found in all eight tumour specimens (fig 2) including the one which showed no fibrous stroma at light microscopy. They could be seen in the perisinusoidal space and between tumour cells as well as in fibrotic regions.

\section{Discussion}

Using both modalities of immunohistochemical and ultrastructural methods, myofibroblasts were identified in hepatitis B associated cirrhosis and hepatocellular carcinoma. They were found in seven of the eight cirrhotic specimens and in all eight tumour specimens. They were identified in the fibrotic areas using an immunohistochemical technique, but ultrastructural examination disclosed their presence in the perisinusoidal space and between tumour cells.

The presence of myofibroblasts has been observed in alcoholic cirrhosis. ${ }^{4}$ They have not been closely studied in other types of cirrhosis or liver disease. Their role in cirrhosis remains to be elucidated, but may be indicative of the basic reparative process that occurs in all types of cirrhosis. In experimentally induced cirrhosis there was evidence that myofibroblasts were derived from the fat storing cells (Ito 


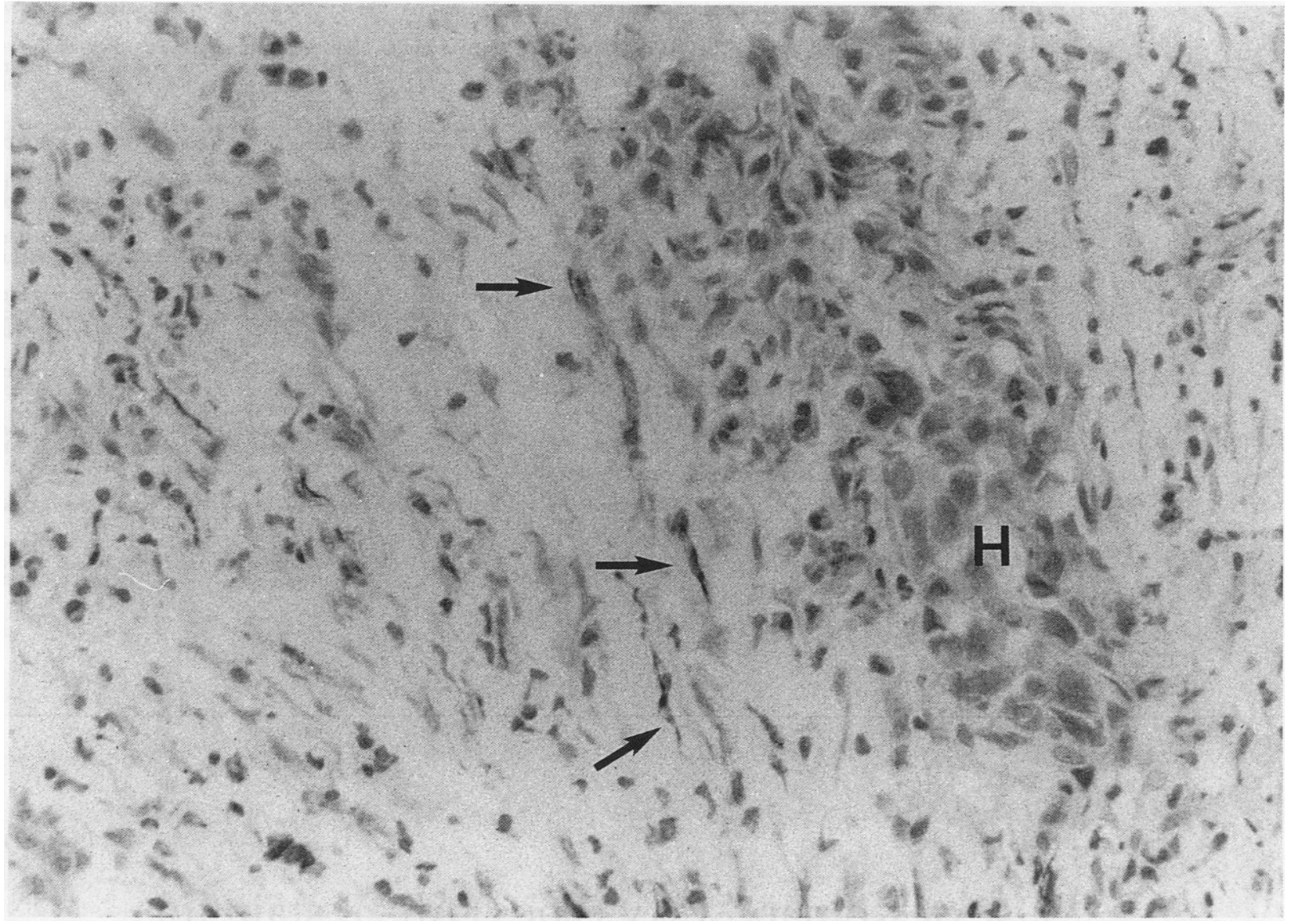

Figure 1 Actin positive stromal cells (arrows) in juxtaposition to a hepatocellular carcinoma $(H)$. Smaller number of these cells are also present in the fibrous septa in the cirrhotic areas (avidin-biotin peroxidase complex).

Figure 2 Myofibroblast in the fibrous stroma of $a$ hepatocellular carcinoma. The actin filaments with dense bodies (D), Golgi apparatus $(G)$, and rough endoplasmic reticulum $(R)$ are clearly seen (TEM)

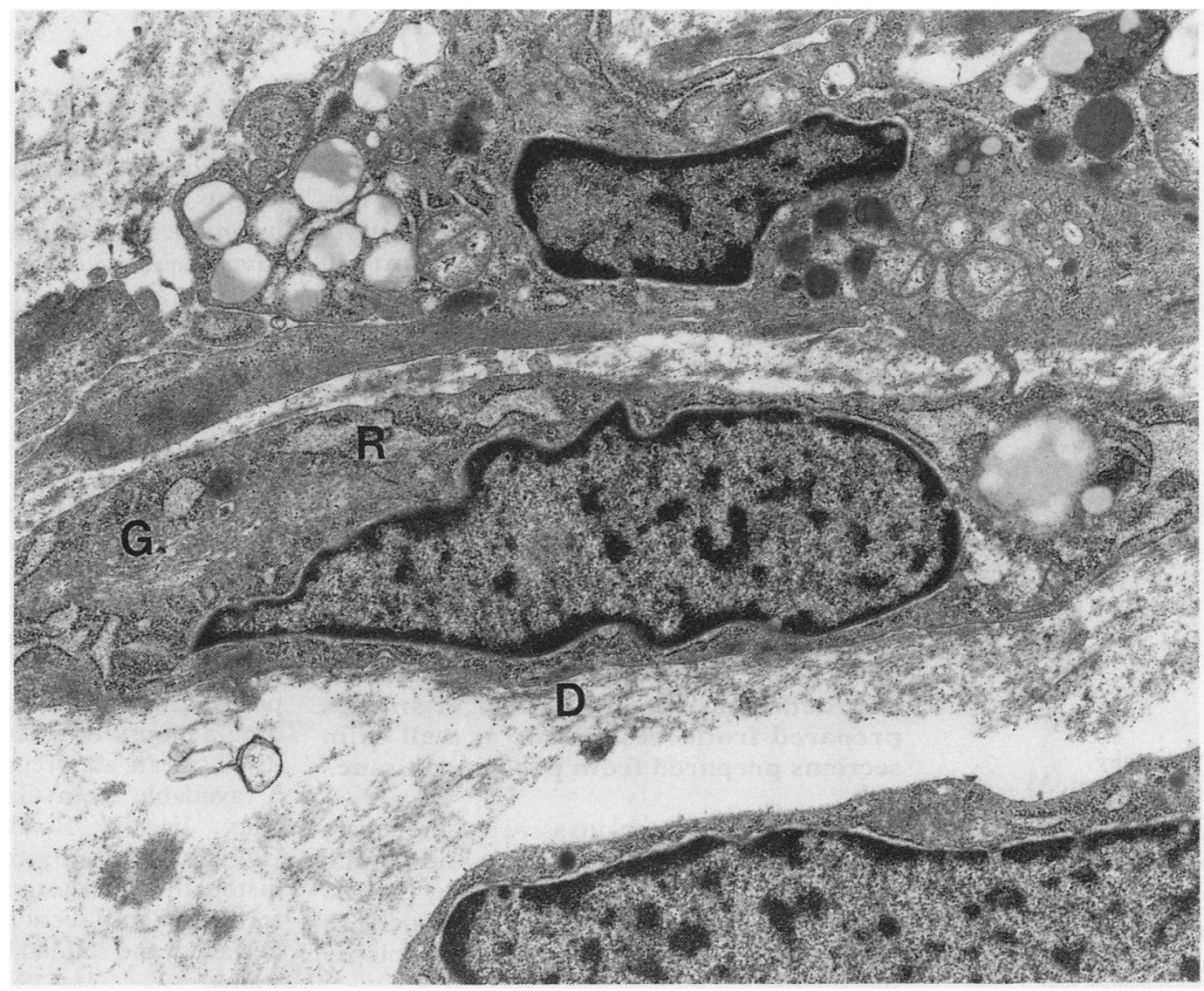


cells), working in concert with activated Kupffer cells, platelets, and regenerating hepatocytes. ${ }^{5}$

A desmoplastic response in hepatocellular carcinoma is not particularly prominent and has not been reported to be related to prognosis. On the other hand, the encapsulated hepatocellular carcinoma and the fibrolamellar carcinoma, which show a distinct stromal reaction, are known to carry a better prognosis. ${ }^{67}$ Evidence suggests that the myofibroblasts have a role in limiting tumour expansion and preventing metastasis by virtue of their physical effect and anticollagenolytic properties. ${ }^{89}$ In a recent report it was suggested that the ability of the hepatocellular carcinoma cells to digest the extracellular matrix was related to tumour aggression. ${ }^{10}$ The presence of myofibroblasts in hepatocellular carcinoma is therefore interesting and warrants further investigation.
1 Ahmed A. The myofibroblast in breast disease. Pathol Annu 1990;2:237-86.

2 Arteaga CL, Tandon AK, Hoff DD. Transforming growth factor beta: Potential antocrine growth inhibitor of oesfrogen receptor negative human breast cancer cells $\mathrm{Can}$ trogen receptor negative
cer Res $1987 ; 48: 3898-904$

3 Tsukes T, McNutt MA, Ross R. HHF35, a muscle actin specific monoclonal antibody. Am J Pathol 1987;127: s89-402.

4 Rudolph R, McClure WJ, Woodward M. Contractile fibroblasts in chronic alcoholic cirrhosis. Gastroenterology 1979;76:704-9.

5 Gressner AM, Bachem MG. Cellular sources of noncollagenous matrix proteins: role of fat-storing cells in fibrogenesis. Semin Liver Dis 1990;10:30-46.

6 Berman MM, Libbey NP, Foster JH. Hepatocellular carcinoma of polygonal cell type with fibrous stroma-an atypical variant with a favourable prognosis. Cancer 1980;46: 1448-55.

7 Hsu HC, Wu TT, Wu MZ: Tumour invasiveness and prognosis in resected hepatocellular carcinoma: clinical prognosis in resected hepatocellular carcinoma: clinical

8 and pathogenetic implications. Cancer 1988;61:2095-9. taneous metastasis of BL6 melanoma with inhibition of the taneous metastasis of BL6 melanoma with inhibition of the desmoplastic res

9 Barsky SH, Nelson LL, Levy VA. Tumour desmoplasia inhibits angiogenesis. Lancet 1987;ii:1336-7.

10 Grigioni WF, Garbisa S, D'Errico A. Evaluation of hepatocellular carcinoma aggressiveness by a panel of extracellular matrix antigens. Am J Pathol 1991;138: 647-54

\title{
Use of Romanowsky type (Diff-3) stain for detecting Helicobacter pylori in smears and tissue sections
}

\author{
A M Zaitoun
}

\begin{abstract}
A Romanowsky type (Diff-3) stain was used for identifying Helicobacter pylori in gastric biopsy specimens from 50 patients with ulcer and non-ulcer dyspepsia. Air dried smears were prepared from fresh biopsy tissue and histological sections were prepared from paraffin wax processed tissue. The Diff-3 technique is accomplished in five steps and takes about 30 seconds. Results using the Diff-3 stain correlated $100 \%$ with those using the Giemsa stain. The Diff-3 stain is reliable, simple, rapid, easy and clean, and smears prepared from fresh biopsy tissue can be examined and an immediate report given. The method is recommended for the identification of $H$ pylori in smears prepared from fresh tissue as well as in sections prepared from processed tissue.
\end{abstract}

Pathology

Qassimi Hospital, PO

Box 3500 Sharjah,

United Arab Emirates

A M Zaitoun

Correspondence to:

Dr A M Zaitoun

Accepted for publication

23 September 1991
Many invasive and non-invasive techniques are currently, used for research into the natural history of Helicobacter pylori. Recent interest has focused on finding a simple and quick test for the detection of $H$ pylori in gastric biopsy specimens. ${ }^{1}$
Histological detection of $H$ pylori in gastric biopsy specimens can be achieved using several techniques, including the Warthin-Starry silver stain, ${ }^{2}$ Giemsa, ${ }^{3}$ half-Gram, ${ }^{4}$ Gimenéz, Cresyl fast violet, ${ }^{6}$ Brown-Hopps ${ }^{7}$ and immunohistochemical methods. ${ }^{8}$

\section{Methods}

Gastric biopsy specimens from 50 patients with ulcer and non-ulcer dyspepsia were studied by histological and cytological techniques for the presence of $H$ pylori. Gastric biopsy specimens received in this laboratory include one antral biopsy used for direct smear, urease test, and culture, and a second biopsy specimen for the preparation of histological sections. After grinding a biopsy fragment in a sterile grinder air dried smears are prepared and stained by Diff3 (available from Gainland Chemical Company, Factory Road, Sandycroft, Deeside, Clwyd, Wales). Gastric biopsy specimens for histopathological examination are processed in formalin, and sections $4 \mu \mathrm{m}$ thick are cut, dewaxed, and stained by the Diff- 3 and Giemsa stains. 